\section{Computational fluid dynamic analysis of calcified coronary plaques: correlation between hemodynamic changes and cardiac image analysis based on left coronary bifurcation angle and lumen assessments}

The purpose of this study was to determine the relationship between left coronary bifurcation angle and significant coronary stenosis with use of coronary CT angiography (CCTA)-generated computational fluid dynamics (CFD) analysis when compared to the CCTA analysis of coronary lumen stenosis with invasive coronary angiography (ICA) as the reference method. Eleven patients with calcified plaques at the left coronary artery tree who underwent CCTA and ICA examinations were included in the study. CFD simulation of left coronary models was performed to analyse hemodynamic changes including wall shear stress, wall pressure and flow velocity. The mean bifurcation angle was measured $83.3 \pm 17.1^{\circ}$ and $83.3 \pm 17.0^{\circ}$ on CCTA and ICA, respectively, with no significant difference $(p=0.99)$. Of 15 significant stenosis at left anterior descending (LAD) and left circumflex (LCX) on CCTA, only 3 of them were confirmed to be $>50 \%$ stenosis on ICA. Wall shear stress was noted to increase in the LAD and LCX models with significant stenosis and wider angulation $\left(>80^{\circ}\right.$ ), but remained no change in most of the other coronary models with no significant stenosis and narrower angulation. Wall pressured was decreased at the significant stenotic lesions, while flow velocity was increased with flow turbulence at the post-stenotic sites. This study further clarifies the direct correlation between left coronary bifurcation angle and significant stenosis, with angulation measurement being more accurate than lumen assessment for diagnosing significant stenosis.

Submitted: 17 October 2016; Accepted: 08 November 2016; Published online: 14 November 2016

Keywords: Angulation - Coronary artery disease - Calcified plaque - Computational fluid dynamics - Correlation

Coronary CT angiography (CCTA) is a widely performed imaging modality for the diagnostic assessment of coronary artery disease. Rapid developments in multi-slice CT scanning techniques enable accurate analysis and characterization of coronary plaque by CCTA [1-3]. The main factor that limits diagnostic performance of CCTA is due to blooming artifact which arises from severe calcification in the coronary artery. This results in high false positive rate, thus, compromising the specificity and positive

\author{
Zhonghua Sun ${ }^{1 *}$, and \\ Thanapong Chaichana \\ 'Department of Medical Radiation \\ Sciences, Curtin University, Perth, \\ Western Australia, 6845, Australia \\ ${ }^{2}$ Biomechanical Electronics Research \\ Group, Department of Mathematics \\ and Computer Science, Liverpool \\ Hope University, Hope Park, Liverpool, \\ England, United Kingdom \\ *Author for correspondence: \\ Tel.: +6189266 7509 \\ Fax: +61892662377 \\ z.sun@curtin.edu.au
}


predictive value of CCTA to a greater extent. It has been reported that in the presence of highly calcified plaques, the specificity of CCTA ranges from $18 \%$ to $53 \%$ based on degree of coronary lumen stenosis [4-8]. To overcome this limitation, strategies have been proposed to improve the specificity including application of image post-processing algorithms to minimize the artifact effect or use of high spatial resolution to improve lumen assessment [9-11]. Using left coronary bifurcation angle to determine significant coronary stenosis is another approach for diagnosis of calcified coronary plaques with promising results reported when compared to the conventional method of coronary lumen assessment $[5,12,13]$.

The rationale of taking into account coronary bifurcation angle for assessment of coronary artery disease is due to the well-known fact that there is close association between hemodynamic changes and atherosclerosis development at bifurcation regions [1417]. Plaque proliferation occurs when wall shear stress is decreased due to increased bifurcation angle or in the vascular areas of abrupt curvatures [14,18]. Wider bifurcation angles have been shown to be associated with higher-risk plaques in comparison with the arteries with narrower angles, highlighting the correlation between bifurcation angle and plaque development $[14,19]$. Despite these studies assessing the bifurcation angle and hemodynamic changes in relation to coronary artery disease or plaque development, to the best of our knowledge, no report is available on the investigation of relationship between bifurcation angle and coronary lumen assessment and corresponding hemodynamics. Thus, the purpose of this study is to further confirm the diagnostic value of using bifurcation angle for assessment of calcified coronary plaques by correlating hemodynamic changes to the coronary artery with degree of coronary stenosis based on CCTA. 3D reconstruction of coronary artery and plaques with numerical simulation using computational fluid dynamics (CFD) based on patient-specific imaging data has been increasingly used in recent years to study hemodynamics in cardiovascular disease [20-22]. We hypothesized that the method of using bifurcation angle with CCTA is closely related to the corresponding hemodynamic changes with CCTA-derived CFD analysis in the diagnosis of calcified plaques.

\section{Materials and Methods}

\section{Study population}

Patients undergoing CCTA and invasive coronary angiography (ICA) for the diagnosis of coronary artery disease were retrospectively reviewed. Only those patients with calcified plaques in the left coronary artery tree detected by CCTA with degree of coronary stenosis confirmed by ICA were included in the study. Out of 40 patients with CCTA and ICA imaging examinations, 11 (7 males, mean age: $62.2 \pm 6.8$ years) were selected for this study, while the remaining patients were excluded due to presence of non-calcified or mixed plaques $(n=20)$, coronary stents $(n=5)$, and poor image quality $(n=4)$.

All patients were scanned with the second-generation of dual-source CT (Somatom Definition Flash, Siemens Healthcare, Forchheim, Germany). The scanning parameters, contrast injection and patient preparation in terms of heart rate control for CCTA protocol and ICA procedure were described previously [5].

All procedures performed in this study were in accordance with the ethical standards of the institutional and/or national research committee. Ethics approval was waived in this study as data acquisition of CCTA comprises routine diagnostic examination of coronary artery disease. Due to the retrospective nature of this study, informed consent was not required from the patients.

\section{Measurement of left coronary bifurcation angle}

Left coronary bifurcation between left anterior descending (LAD) and left circumflex (LCx) was measured on both CCTA and ICA images. Measurements were performed by two investigators (with 8 and more years of experience in cardiac CT imaging) with angle measured during diastolic phases during CCTA and ICA images with the aim of obtaining maximum angulation during this cardiac phase. Three consecutive angle measurements were obtained with the mean value taken as final to avoid intra- and interobserver disagreement. A degree of $>80^{\circ}$ bifurcation angle is used as a cut-off value to determine significant coronary artery disease or stenosis, according to previous studies [13,19,23-25].

\section{Generation of left coronary artery model for CFD analysis}

Segmentation of CCTA volume data was performed with a semiautomatic technique to remove bony and other structures while only extracting the topology of the left coronary artery tree consisting of left main stem, LAD and LCx branches, identifying coronary plaques and segmenting lumen boundary along the left coronary tree. Details of image post-processing and segmentation have been described in previous studies [26,27]. In brief, 3D volume datasets were post-processed on a workstation with Analyze 12.0 (Analyze Direct, Inc., Lexana, KS, USA). Image post-processing and segmentation was based on CT number thresholding technique, with scan-related artifacts and soft tissues being removed in 2D axial and $3 \mathrm{D}$ reconstructed images. The purpose of image 
segmentation was to generate 3D surface models which consist of left main stem coronary artery, LAD and LCx and its main side branches. The $3 \mathrm{D}$ left coronary artery surface models of these cases were saved in "binary STL (stereo-lithography) format" for generation of computational models.

The binary STL files were transferred to a workstation for reconstruction purposes and CFD simulation. Although the original rough surface geometry of left coronary artery was kept, the surface models were smoothed to remove some artifacts (such as non-physical artifacts caused by sharp edges). The computational left coronary artery models were saved into "STL format" for generation of computational elements of the models using ANSYS ICEM CFD version $12 \mathrm{v}$ (ANSYS, Inc., Canonsburg, PA, USA). CFD simulations were conducted with implementation of parameters of realistic physiological boundary conditions in these models, and this has been described in detail in previous studies [14,26,28]. In brief, a time dependent simulation was computed, with the hemodynamic profiles including flow velocity, wall pressure and wall shear stress (WSS) being calculated and compared with findings from image analysis by CCTA and ICA in terms of the bifurcation angle and lumen stenosis.

\section{Generation of medical computing for CFD modelling}

The essential parameters of CFD modelling were mainly chosen to conduct medical image computing for cardiovascular blood flow analysis. The transient flow replicating both systolic and diastolic phases was applied as the boundary flow computation of an inflow condition [14,26]. The parameters of rheological properties were applied as the blood flow was hypothesized to be laminar flow in the small region of blood vessels, the viscosity of the blood was assumed to be 0.0035 Pas, and density of the blood was premised to be $1060 \mathrm{~kg} / \mathrm{m}^{3}$. Blood modelling was assumed to be an incompressible fluid and a Newtonian fluid model. Blood vessel wall modelling was genuinely defined as no-slip condition which was exerted at the walls, and a rigid wall model was assumed to be on a micro millimeter of vascular movement of small vessels. The ANSYS CFX version 12 (ANSYS, Inc.) was used to solve the govern simulation equation as of Navier Stokes equation. Hemodynamic visualisations were analysed during the systolic and diastolic phases for statistical analysis and the presented hemodynamic factors in terms of WSS, wall pressure and flow velocity were chosen effectively to reflect actual effect upon the purpose of the study.

\begin{tabular}{|c|c|c|c|c|c|c|c|c|c|c|}
\hline \multirow{2}{*}{$\begin{array}{l}\text { No. of } \\
\text { Cases }\end{array}$} & \multirow{2}{*}{$\begin{array}{l}\text { Bifurcation } \\
\text { angle on } \\
\left.\text { CCTA ( }{ }^{\circ}\right)\end{array}$} & \multirow{2}{*}{$\begin{array}{c}\text { Bifurcation } \\
\text { angle on } \\
\left.\text { ICA ( }{ }^{\circ}\right)\end{array}$} & \multicolumn{2}{|c|}{$\begin{array}{l}\text { Degree of lumen } \\
\text { stenosis on CCTA }\end{array}$} & \multicolumn{2}{|c|}{$\begin{array}{l}\text { Degree of lumen } \\
\text { stenosis on ICA }\end{array}$} & \multirow[b]{2}{*}{ WSS } & \multicolumn{2}{|l|}{ CFD analysis } & \multirow{2}{*}{$\begin{array}{c}\text { Risk } \\
\text { Assessment } \\
\text { Angulation/ } \\
\text { Lumen }\end{array}$} \\
\hline & & & LAD & LCx & LAD & LCx & & Wall pressure & Flow velocity & \\
\hline 1 & 99 & 99.5 & $74 \%$ & $33 \%$ & $89 \%$ & $25 \%$ & $\begin{array}{l}\text { High at LAD } \\
\text { stenotic site }\end{array}$ & $\begin{array}{l}\text { Decreased at LAD } \\
\text { stenotic site }\end{array}$ & $\begin{array}{l}\text { Increased at LAD } \\
\text { stenotic site }\end{array}$ & $\Delta$ \\
\hline 2 & 78 & 70 & $62 \%$ & - & $44 \%$ & - & $\begin{array}{l}\text { No significant } \\
\text { change }\end{array}$ & $\begin{array}{c}\text { Slightly decreased } \\
\text { at LAD }\end{array}$ & $\begin{array}{c}\text { Slightly turbulent } \\
\text { at LAD }\end{array}$ & $\checkmark$ \\
\hline 4 & 105 & 105 & $72 \%$ & $87 \%$ & $44 \%$ & $77 \%$ & $\begin{array}{l}\text { Increased at LAD } \\
\text { and LCX }\end{array}$ & $\begin{array}{l}\text { Decreased at LCX } \\
\text { stenotic site }\end{array}$ & $\begin{array}{l}\text { High at LCX } \\
\text { stenotic site }\end{array}$ & $\boldsymbol{\nabla}$ \\
\hline 5 & 68.5 & 75 & $72 \%$ & - & $25 \%$ & - & $\begin{array}{l}\text { No significant } \\
\text { change }\end{array}$ & $\begin{array}{l}\text { No significant } \\
\text { change }\end{array}$ & Laminar flow & $\checkmark$ \\
\hline 8 & 76.2 & 77 & $88 \%$ & $68 \%$ & $28 \%$ & $45 \%$ & $\begin{array}{c}\text { Slightly increased } \\
\text { at LM }\end{array}$ & $\begin{array}{l}\text { No significant } \\
\text { change }\end{array}$ & $\begin{array}{l}\text { Slight increased at } \\
\text { LM and LAD }\end{array}$ & $\boldsymbol{\nabla}$ \\
\hline 9 & 91.5 & 85 & $61 \%$ & $49 \%$ & $61 \%$ & $5 \%$ & $\begin{array}{l}\text { High at LAD } \\
\text { stenotic site }\end{array}$ & $\begin{array}{l}\text { No significant } \\
\text { change }\end{array}$ & $\begin{array}{l}\text { Slight increased } \\
\text { at LAD }\end{array}$ & $\Delta$ \\
\hline 10 & 74 & 78 & $57 \%$ & $71 \%$ & $39 \%$ & $48 \%$ & $\begin{array}{l}\text { No significant } \\
\text { change }\end{array}$ & $\begin{array}{l}\text { No significant } \\
\text { change }\end{array}$ & Laminar flow & $\checkmark$ \\
\hline 11 & 71.3 & 70.7 & $51 \%$ & - & $19 \%$ & - & High at LM & $\begin{array}{l}\text { No significant } \\
\text { change }\end{array}$ & $\begin{array}{c}\text { Slightly increased } \\
\text { at LM }\end{array}$ & $\nabla$ \\
\hline
\end{tabular}

CCTA-coronary CT angiography, ICA-invasive coronary angiography, LM-left main stem, LAD-left anterior descending, LCX-left circumflex, WSS-wall shear stress, $\mathbf{\Delta}$ high risk of coronary stenosis and angulation, $\boldsymbol{\nabla}$ low risk of coronary stenosis and angulation, $\checkmark$ normal coronary lumen with no risk of developing significant stenosis. 


\section{Statistical analysis}

Data were entered into SPSS 24.0 for statistical analysis (SPSS Inc, Chicago., IL, USA). Continuous variables were expressed as mean \pm standard deviation, while categorical variables were presented as percentages. Chi-square test was used for analysis of categorical variables or Fisher's Exact test. One sample T test was used to determine the differences of bifurcation angle between CCTA and ICA. A p value of $<0.05$ was considered statistically significant.

\section{Results}

Table 1 shows the distribution of coronary plaques in the left coronary arteries with corresponding bifurcation angle, coronary lumen measurements on CCTA and ICA, as well as the CFD analysis in each case. The mean bifurcation angle measured with CCTA and ICA was $83.3 \pm 17.1^{\circ}$ and $83.3 \pm 17.0^{\circ}$ respectively, with no significant difference between these two modalities ( $\mathrm{p}$ $=0.99$ ). However, coronary stenosis was overestimated in $80 \%$ of the lumen assessment on CCTA, as shown in the table. Significant stenosis $(>50 \%)$ was observed at LAD by CCTA in all of the 11 cases, while only two of them were confirmed to be $>50 \%$ stenosis by ICA. Similarly, of four LCx stenosis of $>50 \%$ on CCTA, only one was confirmed to be significant by ICA. Figures $1 \mathrm{~A}$ and $1 \mathrm{~B}$ are an example of calcified plaques at LAD with overestimated stenosis on CCTA, but ICA shows mild stenosis.

Table 1 also shows the CFD analysis of these patients with findings directly correlated with the bifurcation angle measurement, but not with the degree of lumen stenosis. WSS was noted to increase in the LAD and LCx models with significant stenosis and wider angulation $\left(>80^{\circ}\right)$, but remained no change in most of the coronary models with no significant stenosis or narrower angulation. WSS was slightly increased at the left main (LM) stem regions in

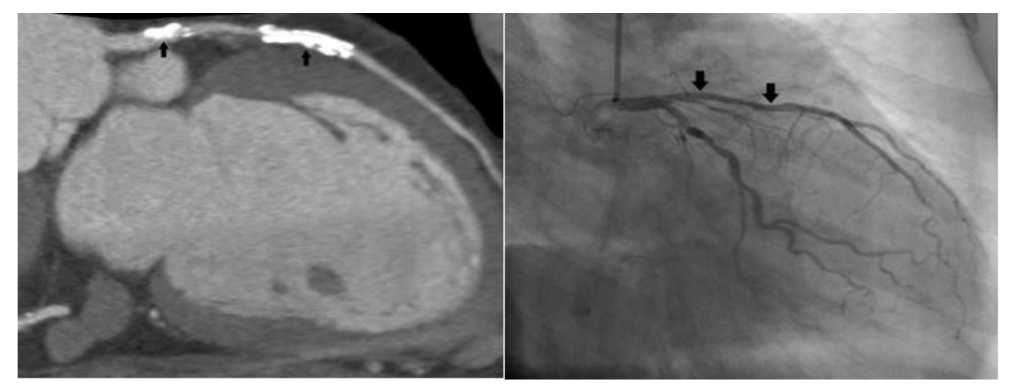

A

Figure 1: Severe calcification in the coronary artery results in overestimation of coronary lumen stenosis. A: Curved planar reformatted coronary CT angiography (CCTA) shows extensively calcified plaques (arrows) at the proximal segment of left anterior descending (LAD) with more than $80 \%$ lumen stenosis. B: Invasive coronary angiography only shows mild stenosis of $<50 \%$ (arrows), indicating the overestimation of degree of stenosis in CCTA. two cases, although there was no significant lumen stenosis on ICA. Wall pressure was decreased in two of the three models with significant coronary stenosis, while flow velocity was increased with post-stenotic flow turbulence in the presence of significant stenosis at the LAD and LCx. Figures $2 \mathrm{~A}-2 \mathrm{C}$ shows CFD modelling of a patient with wide bifurcation angle and significant lumen stenosis at $\mathrm{LAD}$, while Figures $3 \mathrm{~A}-3 \mathrm{C}$ is another example of a narrow bifurcation angle without significant stenosis.

\section{Discussion}

This study is conducted to further clarify the diagnostic value of using bifurcation angle for determination of coronary artery stenosis with use of CFD modelling when compared to the conventional approach of using coronary lumen stenosis. Results showed that there is a direct correlation between left coronary bifurcation angle and corresponding hemodynamic changes with wider angulation and significant stenosis associated with increased WSS and flow velocity and decreased all pressure changes. In contrast, the narrower angulation without significant stenosis led to no changes to the hemodynamic parameters on CFD analysis. Therefore, this study further strengthens the idea that bifurcation angle is more accurate than coronary lumen stenosis for diagnostic assessment of calcified coronary plaques.

The role of wall shear stress on atherosclerosis has been widely studied with low shear stress contributing to plaque formation, while high shear stress leading to plaque rupture [22]. Simulation of coronary flow conditions in a patient-specific coronary model is available with use of CCTA-generated CFD models. Hemodynamic analysis using CFD is considered to be more accurate than CCTA-based lumen analysis for determining significant coronary stenosis. This has been confirmed by several multicentre studies comparing CCTA-generated CFD with CCTA. The DISCOVER-FLOW, DeFACTO and NXT trials are three representative studies showing that CCTAderived fractional flow reserve (FFRCT) is superior to CCTA with improved diagnostic value for diagnosis of ischemic coronary lesions on both per-patient and per-vessel analysis [29-31]. This has significant health and economic implications due to the beneficial effects of using FFRCT as a reliable gatekeeper to invasive procedures and revascularization. These studies also support the use of CFD technique for detection of significant coronary stenosis because the degree of coronary lumen stenosis is not a reliable indicator to determine functional significant of the lesion.

CCTA-generated CFD analysis of coronary hemodynamics has gained increasing attention recently. 
Park et al. in their recent study analysed hemodynamic changes in 80 patients with plaques located at the left coronary artery tree [20]. Higher WSS was found to be at stenotic coronary segments when compared with nonstenotic segments, with WSS providing more valuable information than lumen narrowing in determining adverse plaque characteristics and differentiating highrisk plaques. The study suggests that patient-specific CFD modelling based on CCTA provides insight into elucidating the correlation between hemodynamic changes and plaque features and associated clinical events, thus, improving risk stratification for patients with coronary artery disease. This is consistent with other studies highlighting the association between high WSS and plaque progression and plaque vulnerability $[32,33]$. However, the main limitation of these studies is lack of looking at the calcified plaque with regard to the left coronary bifurcation angle and corresponding CFD analysis.

This study was designed to fill this gap by looking at the left coronary bifurcation angle in relation to the hemodynamic changes as assessed by CCTA-based CFD analysis. Findings are consistent with previous reports indicating less favourable WSS distributions for wider angulated coronary arteries [14,15,34], as well as supporting the previous report of direct correlation between bifurcation angle and intraluminal changes of coronary plaques [35]. However, Beier et al. in their study did not notice significant effect of angulation on the flow dynamic changes to the left coronary artery. Their analysis was based on idealized and patientspecific left coronary artery with different angulations. Their results showed that vessel shape features including tortuosity, diameter or incoming angle produce more impact than bifurcation angle on WSS distribution [36]. Further research is required to clarify the correlation between bifurcation angle and WSS changes in the left coronary artery.

This study has some limitations. First, the sample size is small, despite correlating CCTA findings with ICA which is regarded as the gold standard for lumen assessment. Further, significant stenosis was only confirmed in 3 out of 15 coronary lesions. Studies with inclusion of more patients with significant stenosis and different angulations are needed to verify our results. Second, CFD simulation was conducted under rest condition of cardiac physiology. To represent realistic situation of cardiac cycle, CFD simulation under hyperemia should also be conducted. Finally, we did not analyse plaque characteristics to identify vulnerable plaques as we only included calcified plaques in the

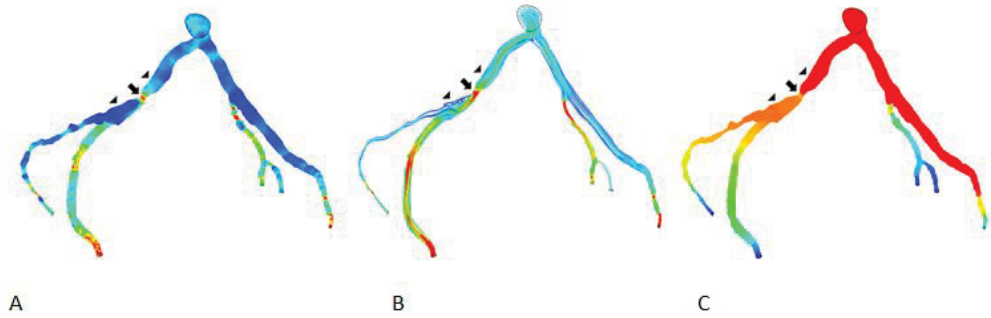

Figure 2: Association between wider angulation and hemodynamic changes by computational fluid dynamics (CFD). Left coronary bifurcation angle between left anterior descending and left circumflex (LCX) was measured $105^{\circ}$ with significant stenosis at LCX on CCTA and ICA in a 58-year-old man. Wall shear stress and flow velocity were increased at the stenotic site of $L C X(A$ and $B)$, while wall pressure was decreased at the same location (C). Arrow refers to the stenotic region at $L C X$, while arrowheads point to the pre- and post-stenotic locations.

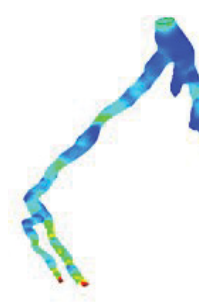

A
Figure 3: Association between narrower angulation and hemodynamic changes by CFD. Left coronary bifurcation angle between left anterior descending (LAD) and left circumflex (LCX) was measured $53^{\circ}$ and $55.7^{\circ}$ on CCTA and ICA in a 65-year-old male, respectively. Significant stenosis ( $>60 \%)$ was noticed at LAD and LCX on CCTA, but no significant stenosis (42-48\%) was confirmed on ICA. There was no significant change to the hemodynamic parameters including wall shear stress, flow velocity and wall pressure $(A-C)$. Arrow refers to the mild stenotic site of LAD, while arrowheads point to the pre- and post-stenotic locations.

left coronary artery tree, since severe calcification is associated with overestimation of coronary lumen stenosis, thus, leading to high false positive rate. Further studies with a focus on plaque features in addition to the bifurcation angle measurement are needed to determine the relationship between angulation and development of high-risk plaques.

\section{Conclusion}

In conclusion, this study shows a direct correlation between wider left coronary bifurcation angle and corresponding hemodynamic changes, mainly the wall shear stress as assessed by CCTA-generated CFD analysis. Results further confirm that coronary bifurcation angle is more accurate than lumen assessment for diagnosing significant stenosis in the presence of calcified plaques. Incorporation of CFD findings into CCTA analysis will add clinical value for differentiating significant stenosis or high-risk plaques in patients with suspected coronary artery disease. Further research on a large cohort with analysis of plaque characteristics is warranted. 
Executive summary

- The purpose of this study was to determine the relationship between left coronary bifurcation angle and significant coronary stenosis with use of coronary CT angiography (CCTA)-generated computational fluid dynamics (CFD) analysis when compared to the CCTA analysis of coronary lumen stenosis with invasive coronary angiography (ICA) as the reference method.

- This study further clarifies the direct correlation between left coronary bifurcation angle and significant stenosis, with angulation measurement being more accurate than lumen assessment for diagnosing significant stenosis.

\section{References}

1. Sara L, Rochitte CE, Lemos PA, et al. Accuracy of multidetector computed tomography for detection of coronary artery stenosis in acute coronary syndrome compared with stable coronary disease: A CORE64 multicenter trial subsidy. Int. J. Cardiol. 177(2), 385-391 (2014).

2. Dey D, Achenbach S, Schuhabeck A, et al. Comparison of quantitative atherosclerotic plaque burden from coronary CT angiography in patients with first acute coronary syndrome and stable coronary artery disease. J. Cardiovasc. Comput. Tomor. 8(5), 368-374 (2014).

3. Villadsen PR, Peterson SE, Dey D, et al. Coronary atherosclerotic plaque burden and composition by $\mathrm{CT}$ angiography in Caucasian and South Asian patients with stable chest pain. Eur. Heart. J. Cardiovasc. Imaging. 25, jew085 (2016).

4. Meng L, Cui L, Cheng Y, Wu X, Tang Y, Xu F. Effect of heart rate and coronary calcification on the diagnostic accuracy of the dual-source CT coronary angiography in patients with suspected coronary artery disease. Korean. J. Radiol. 10(4), 347-354 (2009).

5. Sun Z, Xu L, Fan Z. Coronary CT angiography in calcified coronary plaques: Comparison of diagnostic accuracy between bifurcation angle measurement and coronary lumen assessment for diagnosing significant coronary stenosis. Int. J. Cardiol. 203, 78-86 (2016).

6. Chen CC, Chen CC, Hsieh IC, et al. The effect of calcium score on the diagnostic accuracy of coronary computed tomography angiography. Int. J. Cardiovasc. Imaging. 27(Suppl 1), 37-42 (2011).

7. Park MJ, Jung JI, Choi YS, et al. Coronary CT angiography in patients with high calcium score: evaluation of plaque characteristics and diagnostic accuracy. Int. J. Cardiovasc. Imaging. 27(Suppl 1), 43-51 (2011).

8. Uehara M, Funabashi N, Takaoka H, Fujimoto Y, Kobayashi Y. False-positive findings in 320-slice cardiac CT for detection of severe coronary stenosis in comparison with invasive coronary angiography indicate poor prognosis for occurrence of MACE. Int. J. Cardiol. 172(1), 235-237 (2014).

9. Tanaka R, Yoshioka K, Muranaka K, et al. Improved evaluation of calcified segments on coronary CT angiography: a feasibility study of coronary calcium subtraction. Int. J. Cardiovasc. Imaging. 29(Suppl 2), 75-81 (2013).

10. Sun Z, Ng C, Xu L, Fan Z, Lei J. Coronary CT angiography in heavily calcified coronary arteries: Improvement of coronary lumen visualization and coronary stenosis assessment with image post-processing methods. Medicine. 94(48), e2148 (2015).

11. Pontone G, Bertella E, Mushtaq S, et al. Coronary artery disease: diagnostic accuracy of CT coronary angiography-A comparison of high and standard spatial resolution scanning.
Radiology. 271(13), 688-694 (2014).

12. $\mathrm{Xu} \mathrm{L}$, Sun Z. Coronary CT angiography evaluation of calcified coronary plaques by measurement of left coronary bifurcation angle. Int. J. Cardiol. 182, 229-231 (2015).

13. Sun Z, Cao Y. Multislice CT angiography assessment of left coronary artery: Correlation between bifurcation angle and dimensions and development of coronary artery disease. Eur. J. Radiol. 79(2), e90-e95 (2011).

14. Chaichana T, Sun Z, Jewkes J. Computation of hemodynamics in the left coronary artery with variable angulations. $J$. Biomech. 44(10), 1869-1878 (2011).

15. Markl M, Wegent F, Zech T, et al. In vivo wall shear stress distribution in the carotid artery: Effect of bifurcation geometry, internal carotid artery stenosis, and recanalization therapy. Circ. Cardiovasc. Imaging. 3(6), 647-655 (2010).

16. Arjmandi Tash O, Razavi SE. Numerical investigation of pulsatile blood flow in a bifurcation model with a non-planar branch: The effect of different bifurcation angles and nonplanar branch. Bioimpacts. 2(4), 195-205 (2012).

17. Rodriguez-Granillo GA, Garcia-Garcia HM, Wentzel J, et al. Plaque composition and its relationship with acknowledged shear stress patterns in coronary arteries. J. Am. Coll. Cardiol. 47(4), 884-885 (2006).

18. Katritsis D, Kaiktsis L, Chaniotis A, Pantos J, Efstathopoulos EP, Marmarelis V. Wall shear stress: theoretical considerations and methods of measurement. Prog. Cardiovasc. Dis. 49(5), 307-327 (2007).

19. Temov K, Sun Z. Coronary computed tomography angiography investigation of the association between left main coronary artery bifurcation angle and risk factors of coronary artery disease. Int. J. Cardiovasc. Imaging. 32(1), S129-S137 (2016).

20. Park JB, Choi G, Chun EJ, et al. Computational fluid dynamic measures of wall shear stress are related to coronary lesion characteristics. Heart. 102(20), 1655-1661 (2016).

21. Pang CL, Alcock R, Pilkington N, Reis T, Roobottom C. Determining the haemodynamic significance of arterial stenosis: the relationship between CT angiography, computational fluid dynamics, and non-invasive fractional flow reserve. Clin. Radiol. 71, 750-757 (2016).

22. Sun Z, Xu L. Computational fluid dynamics in coronary artery disease. Comput. Med. Imaging. Graph. 38(8), 6512-6663 (2014).

23. Reig J, Petit M. Main trunk of the left coronary artery: anatomic study of the parameters of clinical interest. Clin. Anat. 17(1), 6-13 (2004).

24. Pflederer T, Ludwig J, Ropers D, Daniel WG, Achenbach S. Measurement of coronary artery bifurcation angles by multidetector computed tomography. Invest. Radiol. 41(11), 793-798 (2006). 
25. Kawasaki T, Koga H, Serikawa T, et al. The bifurcation study using 64 multislice computed tomography. Catheter. Cardiovasc. Interv. 73(5), 653-658 (2009).

26. Chaichana T, Sun Z, Jewkes J. Hemodynamic impacts of various types of stenosis in the left coronary artery bifurcation: A patient-specific analysis. Phys. Med. 29(5), $447-452$ (2013).

27. Chaichana $T$, Sun Z Investigation of the hemodynamic effect of stent wires on renal arteries in patients with abdominal aortic aneurysms treated with suprarenal stent-grafts. Cardiovasc. Intervent. Radiol. 32(4), 647-657 (2009).

28. Shanmugavelayudam SK, Rubenstein DA, Yin W. Effect of geometrical assumptions on numerical modelling of coronary blood flow under normal and disease conditions. ASME. $J$. Biomech. Eng. 132(6), 061004 (2010).

29. Koo BK, Erglis A, Doh JH, et al. Diagnosis of ischemiacausing coronary stenoses by noninvasive fractional flow reserve computed from coronary computed tomographic angiograms. Results from the prospective multicenter discoverflow (diagnosis of ischemia-causing stenoses obtained via noninvasive fractional flow reserve) study. J. Am. Coll. Cardiol. 58(19), 1989-1997 (2011).

30. Min JK, Berman DS, Budoff MJ, et al. Rationale and design of the DeFACTO Determination of Fractional Flow Reserve by Anatomic Computed Tomographic Angiography study. J. Cardiovasc. Comput. Tomogr. 5(5), 301-309 (2011).
31. Norgaard BL, Leipsic J, Gaur S, et al. Diagnostic performance of non-invasive fractional flow reserve derived from coronary CT angiography in suspected coronary artery disease: The NXT trial. J. Am. Coll. Cardiol. 63(12), 1145-1155 (2014).

32. Samady H, Eshtehardi P, McDaniel MC, et al. Coronary artery wall shear stress is associated with progression and transformation of atherosclerotic plaque and arterial remodeling in patients with coronary artery disease. Circulation. 124(7), 779-788 (2011).

33. Gijsen F, van der Giessen A, van der Steen A, Wentzel J. Shear stress and advanced atherosclerosis in human coronary arteries. J. Biomech. 46(2), 240-247 (2013).

34. Dong J, Sun Z, Inthavong K, Tu J. Fluid-structure interaction analysis of the left coronary artery with variable branch angulation. Comput. Med. Biomech. Biomed. Eng. 18(14), 1500-1508 (2015).

35. Sun Z. Coronary CT angiography in coronary artery disease: correlation between virtual intravascular endoscopic appearances and left bifurcation angulation and coronary plaques. Biomed. Res. Int. 2013, 732059 (2013).

36. Beier S, Ormiston J, Webster M, et al. Impact of bifurcation angle and other anatomical characteristics on blood flow-A computational study of non-stented and stented coronary arteries. J. Biomech. 49(9), 1570-1582 (2016). 